\title{
Umbilical Coiling Index- UCl by USG and its Postnatal Correlation
}

\author{
N. Hephzibah Kirubamani ${ }^{1 *}$ and M. R. Meenatshi ${ }^{2}$ \\ 'Department of Obstetrics and Gynaecology, Saveetha Medical College and Hospital, Saveetha University, \\ Thandalam, Chennai-602105, Tamil Nadu, India; hepsi1002@yahoo.co.in \\ ${ }^{2}$ Saveetha Medical College and Hospital, Thandalam, Chennai - 602105, Tamil Nadu, India; \\ meena.subbiah16@gmail.com
}

\begin{abstract}
It was thought that Umbilical Cord Coiling plays an important role in protecting the umbilical cord from external pressure such as tension, pressure, stretching or entanglement. The mechanism of coiling still remains undetermined. According to several studies abnormal umbilical coiling is associated with poor perinatal outcomes. Objectives: To evaluate the sonographic accuracy in determining Umbilical Coiling Index (UCI). To correlate it with postnatal examination of umbilical coiling and its association with obstetric and perinatal outcome. Material and Methods: Prospective observational study was conducted at Saveetha Medical College Obstetrics Department. For 200 pregnant women USG umbilical coiling index was measured during routine foetal evaluation between 28 to 40 weeks. USG-Umbilical Coiling Index was correlated with postnatal umbilical coiling index and its association with obstetric\& perinatal outcome was calculated. Sonologist was blinded for pregnancy outcome. Results: USG -UCI was normal in 52\%, hypocoiling $12 \%$ and hypercoiling $36 \%$.In normal coiling there was no antenatal or intranatal problems. In hypocoiling Spontaneous Preterm Delivery was $50 \%$ and Low Birth Weight was38.9\%, FGR 11.1\%. In hypercoiling group Oligohydramnios was $14.8 \%$, Intrapartum fetal distress33.3\%, Meconium staining of liquor $16.6 \%$. In normal coiling all of them delivered naturally. In hypocoiling and in hypercoiling mode of delivery either forceps $7 \%$ or LSCS $41 \%$. ICU admission in hypocoiling was $18 \%$ and in hypercoiling 3\%. Perinatal mortality was NIL. USG-Umbilical Coiling Index Sensitivity 97.8 specificity, $62.5 \%$, Positive predictive value $96.77 \%$ Negative predictive value $71.4 \%$. Conclusion: In our study there is a good correlation between USG-UCI and postnatal UCI. Our study confirms that there is an adverse perinatal outcome with both hypocoiling and hyper coiling of the umbilical cord.
\end{abstract}

Keywords: Hypocoiling, Hypercoiling, Perinatal Outcome, Umbilical Cord, Umbilical Cord Coiling, UCI-Umbilical Coiling Index

\section{Introduction}

Communication between the placenta and the foetus are through the umbilical cord which provides major foeto maternal unit. Umbilical coil prevents compression, tension and torsion and provides uninterrupted blood supply to the foetus. The umbilical cord is vital to the development, well-being, and survival of the foetus, but is vulnerable to kinking, compressions, traction, and torsion which may affect the perinatal outcome. Wharton's jelly, the amniotic fluid, and the helical patterns of coiling of the umbilical cord protect blood vessel in the umbilical cord. Various hypotheses regarding origin of umbilical cord coiling are there but the exact cause is unknown. Foetal movements, torsion of the embryo, differential umbilical vascular growth rates, fetal hemodynamic forces, and the

${ }^{*}$ Author for correspondence 
arrangement of muscular fibers in the umbilical arterial wall were the hypotheses. The most interesting and mysterious characteristic of the umbilical cord is it's twisted and spiral course of its component blood vessels. As early as 28 days after conception coiling of the umbilical vessels develops and its helices maybe seen at 7 weeks in $95 \%$ of fetuses by ultra sonographic examination. Studies have shown that abnormal umbilical cord coiling is associated with adverse pregnancy outcome. Preterm delivery, fetal death, fetal growth restriction, fetal chromosomal or structural abnormalities, operative delivery for fetal distress, and meconium staining of amniotic fluid is associated with abnormal coiling. Umbilical coiling index can be obtained both antenatally by USG and postnatally by measuring the cord.

\section{Aim}

- To evaluate the sonographic accuracy in determining the Umbilical Coiling Index (UCI) during routine foetal anatomic survey in the second and third trimester and to determine the type of umbilical coiling index.

- To correlate USG-UCI and Postnatal UCI.

- To investigate the association between umbilical cord coiling and obstetric management and perinatal outcome.

\section{Materials and Method}

Prospective observational study was conducted at Saveetha Medical College Obstetrics department. For two hundred consecutive women with singleton pregnancies without gross foetal anomaly, attending antenatal OPD, umbilical coiling index was measured by sonography by the Sonologist, single operator (was blinded about the study) in their late second or third trimester (28 to 40 weeks) during routine foetal anatomic survey. Antenatal UCI (aUCI) was calculated by measuring the distance between 2 pairs of coils from the longitudinal images of the umbilical cord from the inner edge of the artery to the outer edge of the same artery at the adjacent umbilical twist along the ipsilateral cord. UCI was calculated as a reciprocal value of the distance between a pair of coils is shown in Figure 1. Normal umbilical cord coiling is approximately 1 coil $/ 5 \mathrm{~cm}$ of umbilical cord length or 0.20 to $0.24 \mathrm{coils} / \mathrm{cm}$. Under coiling was defined as less than
0.07 coils/cm is shown in Figure 3 and Figure 4 and over coiling as more than $0.30 \mathrm{coils} / \mathrm{cm}$ Figure 2 and Figure 5. Antenatal UCI was compared with postnatal true UCI. The postnatal UCI is calculated by dividing the total number of coils by the total length of the cord in $\mathrm{cm}$, immediately after delivery. The distance from the placental edge to the insertion of the umbilical cord was measured after delivery is shown in Figure 6. All patients were followed up till delivery. The following details, namely gestational age at delivery, parity, maternal age, obstetric history, Apgar scores, meconium staining of the amniotic fluid, birth weight of the neonate, preterm delivery, instrumental delivery for fetal distress, small for dates infants, large for dates infants, anatomic abnormalities, fetal death were noted.

Inclusion Criteria: Normal pregnancy with singleton.

Exclusion Criteria: Maternal medical disorders, Oligohydramnios, Polyhydramnios.

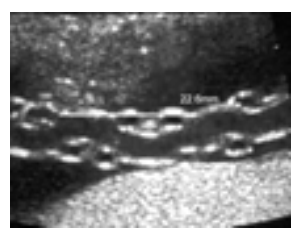

Figure 1. USG-measurement.

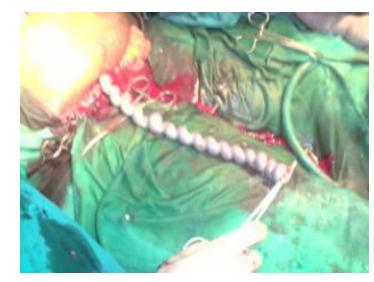

Figure 2. Hyper coiling.

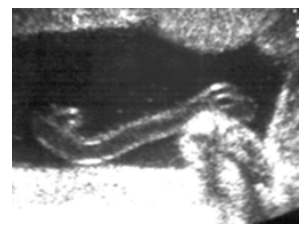

Figure 3. USG-hypocoiling.

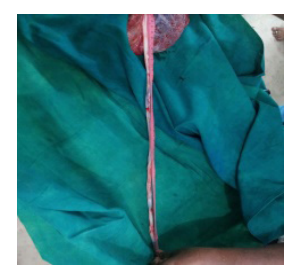

Figure 4. Hypo coiling. 


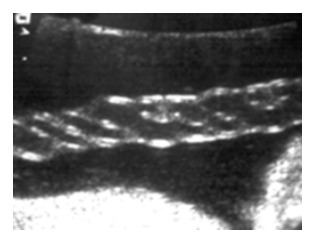

Figure 5. USG-hypercoiling.

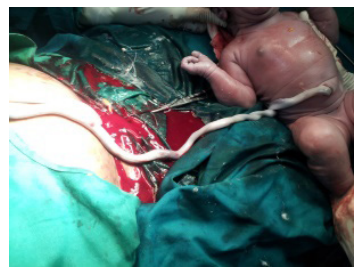

Figure 6. Postnatal measurement.

\section{Results}

We observed 52\% had Normal coiling,12\% had hypocoiling and $36 \%$ had hypercoiling. In Hypocoiling group Spontaneous Preterm Delivery was 50\%, Low Birth Weight was 38.9\%, FGR 11.1\%. In hypercoiling group, Oligohydramnios was $14.8 \%$, Intrapartum fetal distress $33.3 \%$, Meconium staining of liquor 16.6\%. Mode of delivery in normal coiling was natural delivery, inhypocoiling and hypercoiling mode of delivery was either forceps $7 \%$ or LSCS $41 \%$. Perinatal outcome was evaluated and in hypocoiling 18\% required NICU admission for Preterm birth, Low birth weight \& FGR and in hypercoiling group had meconium stained liquor in 3\% and required admission. Perinatal mortality was NIL. In our study USG -Umbilical Coiling Index Specificity 62.5\%, sensitivity 97/8\%, Positive predictive value $96.77 \%$, Negative predictive value $71.4 \%$.

\section{Discussion}

In our study hypocoiling was associated with preterm birth which is similar to the study by ${ }^{1}$. Preterm birth was associated with hypocoiling in our study which is comparablewith ${ }^{2}$. $\operatorname{In}^{3}$ stated in their study that Interventional deliveries and meconium-stained amniotic fluid in labor were observed high in abnormal coiling which is similar to our study. Prevalence of IUGR and non-reassuring fetal heart rates in labor were associated with antenatal USG abnormal coiling in our study and similar statementdoneby ${ }^{\underline{3}}$. Hence this can be used as an indicator for adverse perinatal outcome. $\operatorname{In}^{4}$ concluded that antenatal foetus at risk can be predicted, since UCI has a strong relationship with perinatal outcome. In our study there was no adverse perinatal outcome but all newborn with hypocoiling and $2.7 \%$ newborn with hypercoiling were admitted in NICU. In ${ }^{5}$ study showed more NICU admission in hypocoiled which is similar to our study. In ${ }^{6}$ stated that preterm labour was associated with hypocoiling and foetal heart variability was associated with hypercoiling which correlates with our study. He also concluded that any form of abnormal coiling is associated with adverse neonatal outcome.

\section{Conclusion}

Coiling pattern of umbilical cord visualized by sonography has a potential value in second trimester screening. In the present study, a sincere effort has been made to study the relationship between abnormal coiling of umbilical cord and adverse pregnancy outcome so that in the near future, a prediction for the same can be made, and appropriate preventive measures taken so that every pregnancy ends in a healthy mother and a healthy baby.

\section{Acknowledgement}

The authors thank Chancellor, Vice chancellor, Director and Dean, of Saveetha medical college for the encouragement and for giving me permission to do the study.

\section{References}

1. De Laat MW, Franx A, Bots ML, Visser GH, Nikkels PG. Umbilical coiling index in normal and complicated pregnancies. Obstet Gynecol. 2006 May; 107(5):1049-55. Crossref PMid:16648410

2. Sharma B, Bhardwaj N, Gupta S, Gupta PK, Verma A, Malviya $\mathrm{K}$. Association of umbilical coiling index by colour doppler ultrasonography at 18-22 weeks of gestation and perinatal outcome, J Obstet Gynaecol India. 2012 Dec; 62(6):650-4. Crossref PMid:24293842 PMCid:PMC3575901

3. Predanic M, Perni SC, Chasen ST, Baergen RN, Chervenak FA, Ultrasound evaluation of abnormal umbilical cord coiling in second trimester of gestation in association with adverse pregnancy outcome. Am J Obstet Gynecol. 2005 Aug; 193(2):387-94. Crossref PMid:16098860

4. Ercal T, Lacin S, Altunyurt S, Saygili U, Cinar O, Awe M. Umbilical coiling index: Is it a marker for the foetus at risk? Br J Clin Pract. 1996 Jul-Aug; 50(5):254-6. PMid:8794602 
5. Patil NS, Kulkarni SR, Lohitashwa R. Umbilical cord coiling index and perinatal outcome. J Clin Diagn Res. 2013 Aug; 7(8):1675-7. Crossref
6. Mittal A, Nanda S, Sen J. Antenatal umbilical coiling index as a predictor of perinatal outcome. Arch Gynecol Obstet. 2015 Apr; 291(4):763-8. Crossref PMid:25209352 